\title{
Clinical Application of Bone Morphogenetic Protein-2 Microcarriers Fabricated by the Cryopolymerization of Gelatin Methacrylate for the Treatment of Radial Fracture in Two Dogs
}

\author{
JUEUN PARK ${ }^{1}$, SONG KWON ${ }^{2}$, NATHANIEL S. HWANG ${ }^{2}$ and BYUNG-JAE KANG ${ }^{1}$ \\ ${ }^{1}$ Department of Veterinary Surgery, College of Veterinary Medicine and Institute of Veterinary Science, \\ Kangwon National University, Chuncheon, Republic of Korea; \\ ${ }^{2}$ School of Chemical and Biological Engineering, Institute of Chemical Processes, \\ Seoul National University, Seoul, Republic of Korea
}

\begin{abstract}
Bone morphogenetic protein-2 (BMP-2) effectively induces bone healing. However, the efficacy of $B M P-2$ relies heavily on its delivery vehicle because of its short half-life. We utilized a microcarrier fabricated by the cryopolymerization of gelatin methacrylate (cryoGelMA) infused with bone morphogenetic protein-2 (cryoGelMA$B M P-2)$ for the sustained and localized release of growth factors. Two dogs with radius and ulnar fractures were treated with implanted cryoGelMA-BMP-2 to accelerate bone healing. The cases were followed up for 6 months and 2 months after surgery, respectively. Distinctive healing processes were observed. The operated limb regained its premorbid function, the fracture line disappeared, and the gait was functionally stable. Implantation of cryoGelMA$B M P-2$ resulted in the successful healing of bone fractures.
\end{abstract}

The efficacy of bone morphogenetic protein-2 (BMP-2) application has been demonstrated in various medical fields. One study showed that BMP-2 induced mineralized, osteodentin-like tissue formation at the amputated pulp after 70 days of application (1). Another study demonstrated that BMP-2 application regenerated the cartilage of the trachea in a canine model (2). Additionally, BMP-2 has been shown to play a role throughout the process of bone healing (3). It was

This article is freely accessible online.

Correspondence to: Prof. Byung-Jae Kang, Department of Veterinary Surgery, College of Veterinary Medicine and Institute of Veterinary Science, Kangwon National University, Chuncheon 24341, Republic of Korea. Tel +82 332508207, Fax +82 332595625, e-mail: bjkang@kangwon.ac.kr

Key Words: Bone morphogenetic protein-2, cryopolymerization, dog, gelatin methacrylate hydrogel, radial fracture. also observed that the addition of BMP-2 also induced heterotopic and orthotopic bone formation in rodents that underwent regional therapy (4). Moreover, the implantation of a recombinant human BMP-2 in a synthetic, biodegradable polymer carrier in dogs with proximal femoral bone defects showed bone mass repair at the defect region (5). Nonetheless, despite the importance of BMP-2 in bony tissue development and regeneration, its use has several limitations. For instance, due to its short half-life and poor distribution in vivo, repeated large dose injections are inevitable (6). Furthermore, the unreasonably high cost of continuous application makes it difficult for the wider application of BMP-2 to companion animals (7). As a consequence of these limitations, numerous studies are investigating the use of potential carriers to facilitate a slow and constitutive release of BMP-2. In many cases, gelatin hydrogel is generally used as a carrier for the delivery of biomolecules, such as BMP-2, because it has specific chemical interactions with the agent, and confers an electronegative charge in vivo (8-10). This feature effectively controls the release of biomolecules $(10,11)$. Traditional gelatin is composed of water-in-oil emulsion, and gelatin microspheres that consist of glutaraldehyde $(9,12)$ or genipin $(13,14)$. In this case, we used a microcarrier fabricated by the cryopolymerization of gelatin methacrylate (cryoGelMA), which is the cryogelation product of existing gelatin methacrylate (GelMA). Cryogelation is a reasonably priced and easy method to form macro-pores to accommodate biomolecules, such as growth factor (15). In addition to the existing advantages of GelMA, CryoGelMA is also expected to accommodate higher concentrations of biomolecules, compared to GelMA. GelMA hydrogel has been documented to reduce cellular toxicity and has a wider limit of crosslinking densities compared to that of glutaraldehyde microparticles (16). The broad range of densities for GelMA changes the capacity of the biomolecular loading limit. For instance, GelMA could load 10 times more growth factor than 


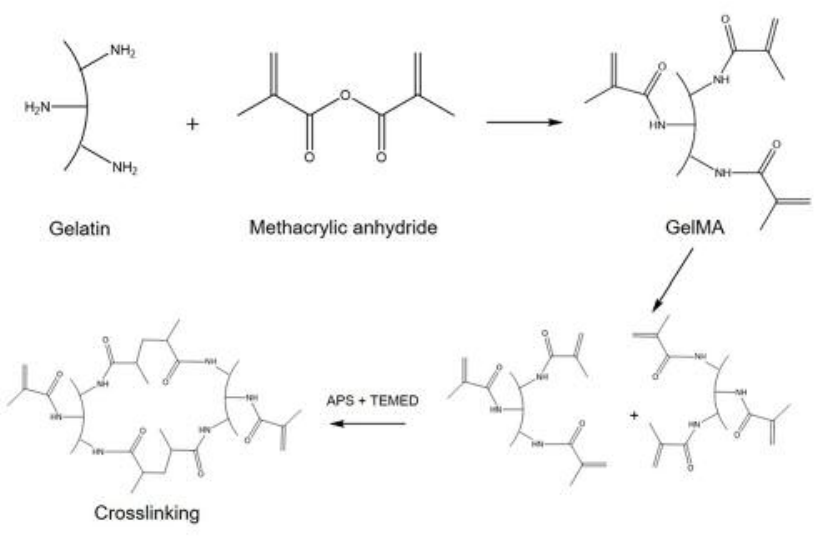

a

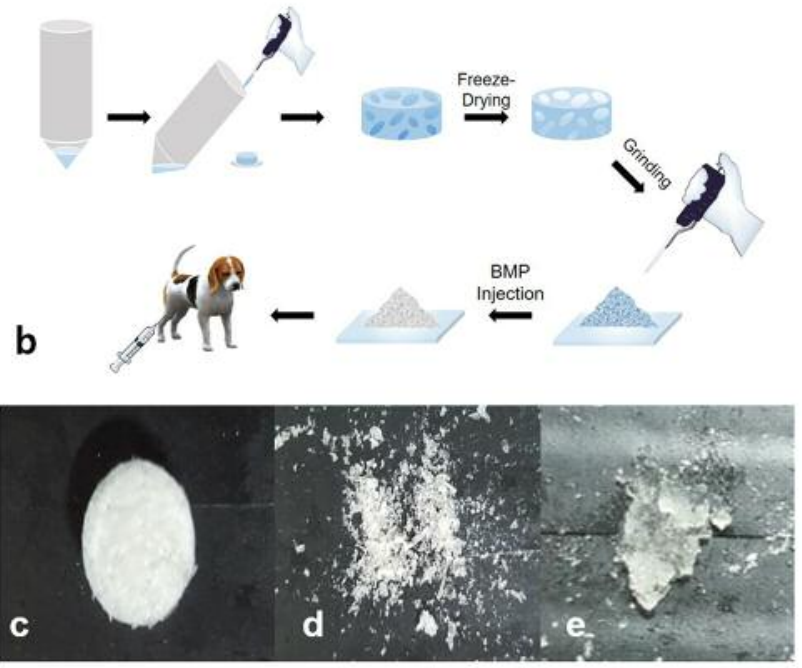

Figure 1. Preparation of cryoGelMA. (a) The synthesis scheme of cryoGelMA, (b) fabrication scheme cryoGelMA-BMP-2, (c) macroscopic images of cryoGelMA, (d) dried cryogel powder, and (e) swell cryogel powder.

that of traditional gelatin (17). The structure of methacrylation directly links to the component ratio of gelatin/particle; this allows for extensive control over the disintegration kinetics. We report the treatment outcomes of two dogs with radial fractures, which were treated with cryoGelMA-BMP-2; in this case, CryoGelMA was used as a carrier of BMP-2 molecules.

We used cryoGelMA and incorporated $0.25 \mathrm{mg}$ of BMP-2 (Anibog ${ }^{\circledR}$, BIOALPHA). We fabricated cryoGelMA and then grinded it into small pieces (Figure 1). In brief, methacrylated gelatins were synthesized by dissolving a porcine skin gelatin into phosphate-buffered saline at $10 \% \mathrm{w} / \mathrm{v}$ at $60^{\circ} \mathrm{C}$. Then, $8 \%$ (v/v) methacrylic anhydride was added at $0.5 \mathrm{ml} / \mathrm{min}$, and the reaction mixtures were reacted at $50^{\circ} \mathrm{C}$ for 3 hours to form the GelMA solution. The GelMA solution was dialyzed against distilled water for 1 week at $40^{\circ} \mathrm{C}$ to remove salts and residues. Cryopolymerization of GelMA was performed with ammonium persulfate (APS) as the initiator agent and N,N,N',N' tetramethylethylenediamine (TEMED) was used as a catalyst. To start the cryopolymerization, $10 \%$ w/v GelMA solution with $4 \%(\mathrm{v} / \mathrm{v})$ APS and $0.2 \%(\mathrm{v} / \mathrm{v})$ TEMED was poured into a cylindrical-shaped polyethylene mold, and placed in a $-20^{\circ} \mathrm{C}$ freezer. Interconnected, macroporous cryoGelMA was then lyophilized, sterilized with UV, and grounded into smaller pieces. The CryoGelMA micro-carriers were then loaded with BMP-2 by immersing the micro-carriers with BMP-2 solution.

\section{Case Report}

Dog 1 (a 5-month-old, $1.85 \mathrm{~kg}$, intact female Pomeranian) was referred to the Kangwon National University veterinary teaching hospital due to its right distal radius and ulnar fracture. The dog had the fracture of the same bone 2 months prior, which was not fixed at all, but the bone was healed with mild bone angulation. After three days of injury, an assigned surgical operation was performed. During surgery, the dog was positioned ventrodorsally, with a craniomedial skin incision performed. There was evidence of soft tissue and bony tissue adhesion, which may have been due to a past fracture history. After exposure of the fracture site, the fracture was reduced by tenting the ends of the bone dorsally, and levering the bone back into its original position. A T-shaped 1.2 locking plate with seven 1.2 locking screws was used on the fractured site for the fixation, and cryoGelMA-BMP-2 was added to the lesion to minimize the possibility of the nonunion, and lead to the enhancement of the healing process (Figures 2 and 3).

Ten days following surgery, some degree of hardened and swollen soft tissue formation was observed around the surgical site. The opacity of the fractured site and the thickness of the ulna were increased (Figure 3c). The swelling of the soft tissue was relieved, and the callus had been developed around the fractured site 3 weeks after surgery (Figure 3d). After 6 weeks, radiography indicated that the callus around the fractured site showed more progressed remodeling than that of the previous image; the fracture line disappeared. Three months after the surgery, the swollen leg almost returned to normal size. Ambulation and weight-bearing returned to its normal condition (Figure 3e). We hypothesized that the swollen and hardening of soft tissue was induced by the application cryoGelMA-BMP-2. After 6 months, remodeling was almost completed, and there was improved stability. Furthermore, the ambulation and weight-bearing conditions of the dog were mostly fine (Figure 3f). 

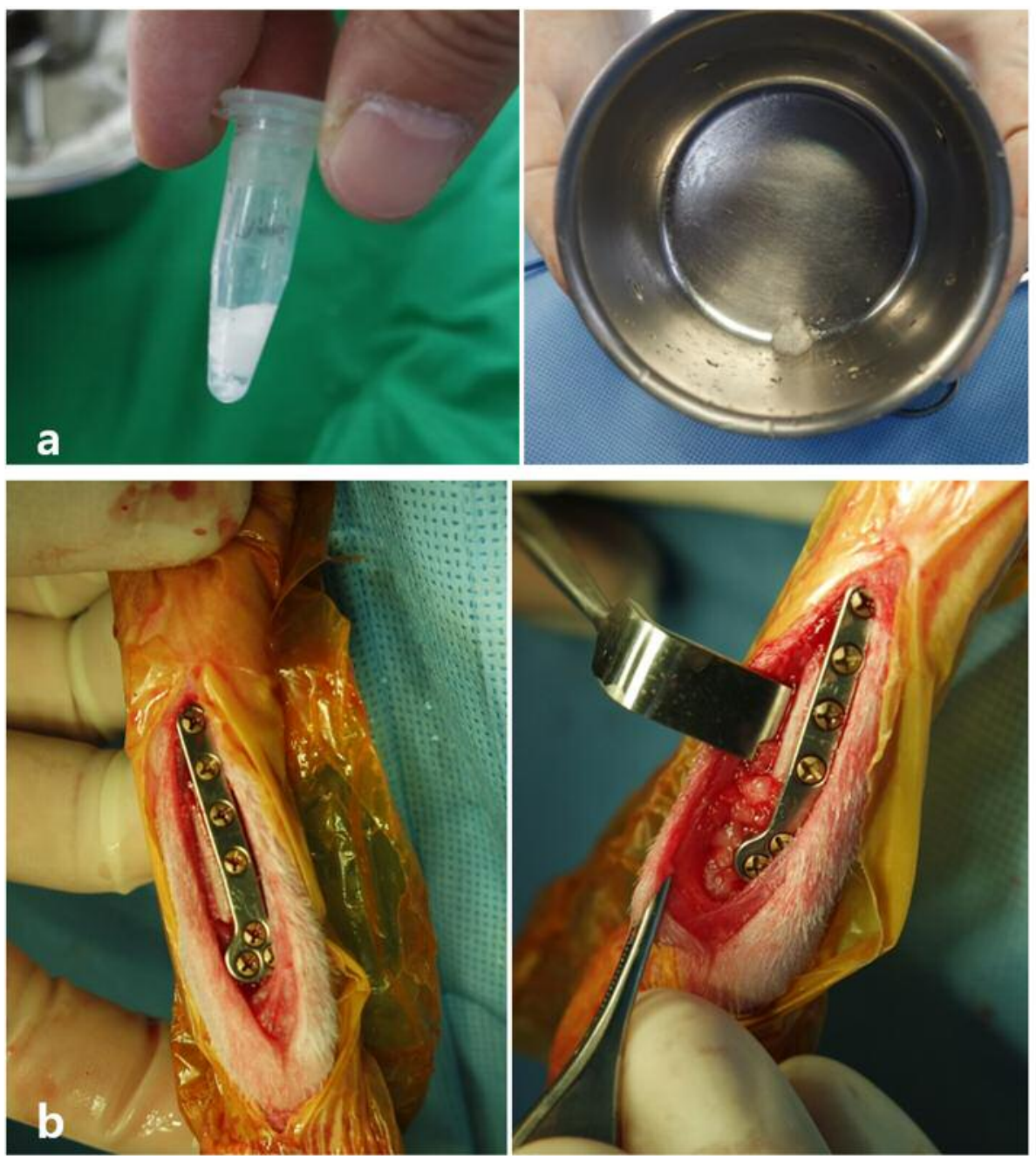

Figure 2. Application of cryoGelMA-BMP-2 at the fractured site. (a) Gross appearance of cryoGelMA-BMP-2 and (b) cryoGelMA-BMP-2 used on the fractured site for the fixation.

Dog 2 (A 1-year-old, $2.7 \mathrm{~kg}$, intact female Poodle) was referred to Kangwon National University veterinary teaching hospital due to its left distal radius and ulnar nonunion fracture. The dog had the fracture 1 year prior, which was fixed by external skeletal fixation; the bone was recovered properly. However, after 6 months, the incidence of refracturing was reported. As the owner was reluctant to surgically fix the fracture, it was managed by splint and bandage. After 4 months since the refracture, radiography showed that bone opacity decreased, and that the shaft was irregular with the continuous presence of the fractured line (Figure 4a). The fracture failed to heal appropriately, and a nonunion was developed. The surgical approach employed for the previous case was similarly performed for this case. Additionally, we used the same cryoGelMA-BMP-2 product.
During surgery, we attempted to lever the bone fragment for the reduction since tissue fibrosis developed at the site, and the alignment of the bone fragment was not accurate. We applied cryoGelMA-BMP-2 between the plate and the radius (Figure 4b). A week after the operation, the margin of the distal fragment was slightly convex shaped. The soft tissue around the site of cryoGelMA-BMP-2 application was swollen and hardened, showing a resemblance to the treatment outcome of the first case (Figure 4c). Bone healing and a narrowed fracture line were observed after 3 weeks of post-operation. Fortunately, the bone activity was positive (Figure 4d). Seven weeks after the operation, the fractured space was remarkably decreased (Figure 4e). Two months after surgery, successfully united bone fragments were observed (Figure 4f). 

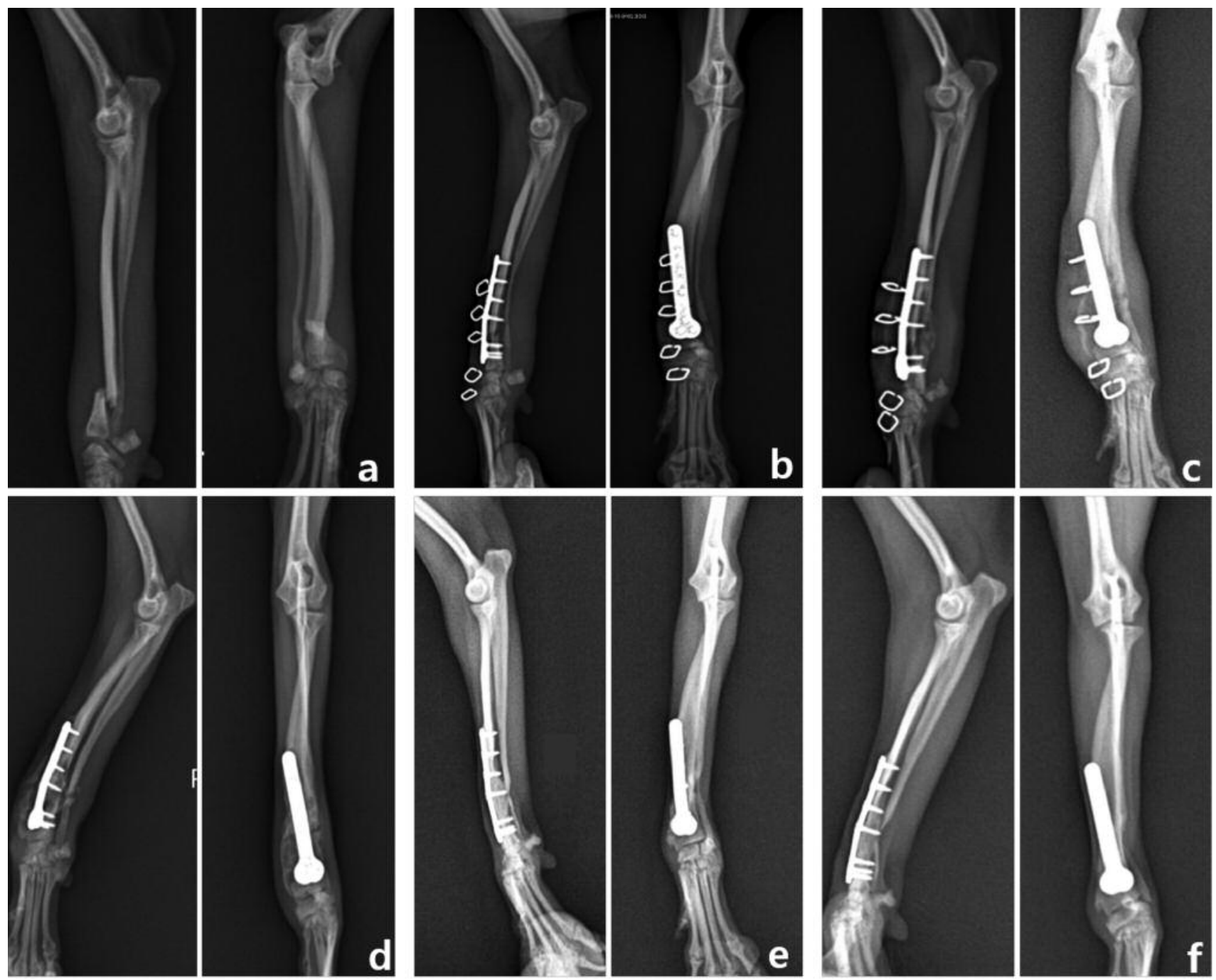

Figure 3. The bone healing process after application of the plate and cryoGelMA-BMP-2 at the fractured site (Lateral, Dorsoventral position). (a) The fracture of the right distal radius and ulna is observed 1 day after the accident. (b) The fracture was surgically fixed by the plate, and of cryoGelMA-BMP-2 was applied. (c) Ten days after surgery, some degree of hardened and swollen soft tissue formation was observed around the surgical site. (d) Three weeks after surgery, the callus had been developed around the fracture site. (e) After 3 months, the fracture site showed more remodeling, and the fracture line disappeared. $(f)$ After 6 months, remodeling was almost completed, and the stability of the site improved.

\section{Discussion}

In both cases, cryoGelMA-BMP-2 was used to prevent nonunion. Although the rigid plate fixation was used in this case, the use of cryoGelMA-BMP-2 induced indirect healing at the site, in addition to excessive callus formation. During bone fracture recovery, no complications, such as heterotopic ossification, were observed. In one study, gelatin hydrogel infused with BMP-2, was used as treatment for a canine orbital floor defect; thick trabeculae with a narrower intertrabecular distance at the complex were observed at the applied site. From the structural analysis, mature trabeculae were observed at the site of the newly formed bone, which was similar to normal bone tissue (8).

The main objective of this study was to demonstrate the efficacy of cryoGelMA-BMP-2. Unlike the injection of BMP-2 solution, or the incorporation of BMP-2 into gelatin hydrogel, GelMA-BMP-2 constitutively releases the same dose of BMP-2 over a prolonged period of time (18). GelMA produces polyionic compounds with loaded growth factor such as BMP-2. Therefore, BMP-2 is charged positively, and gelatin type B is charged negatively, allowing for an interaction between BMP-2 and GelMA. This decelerates the release of the biomolecule $(9,10)$. The release velocity of 

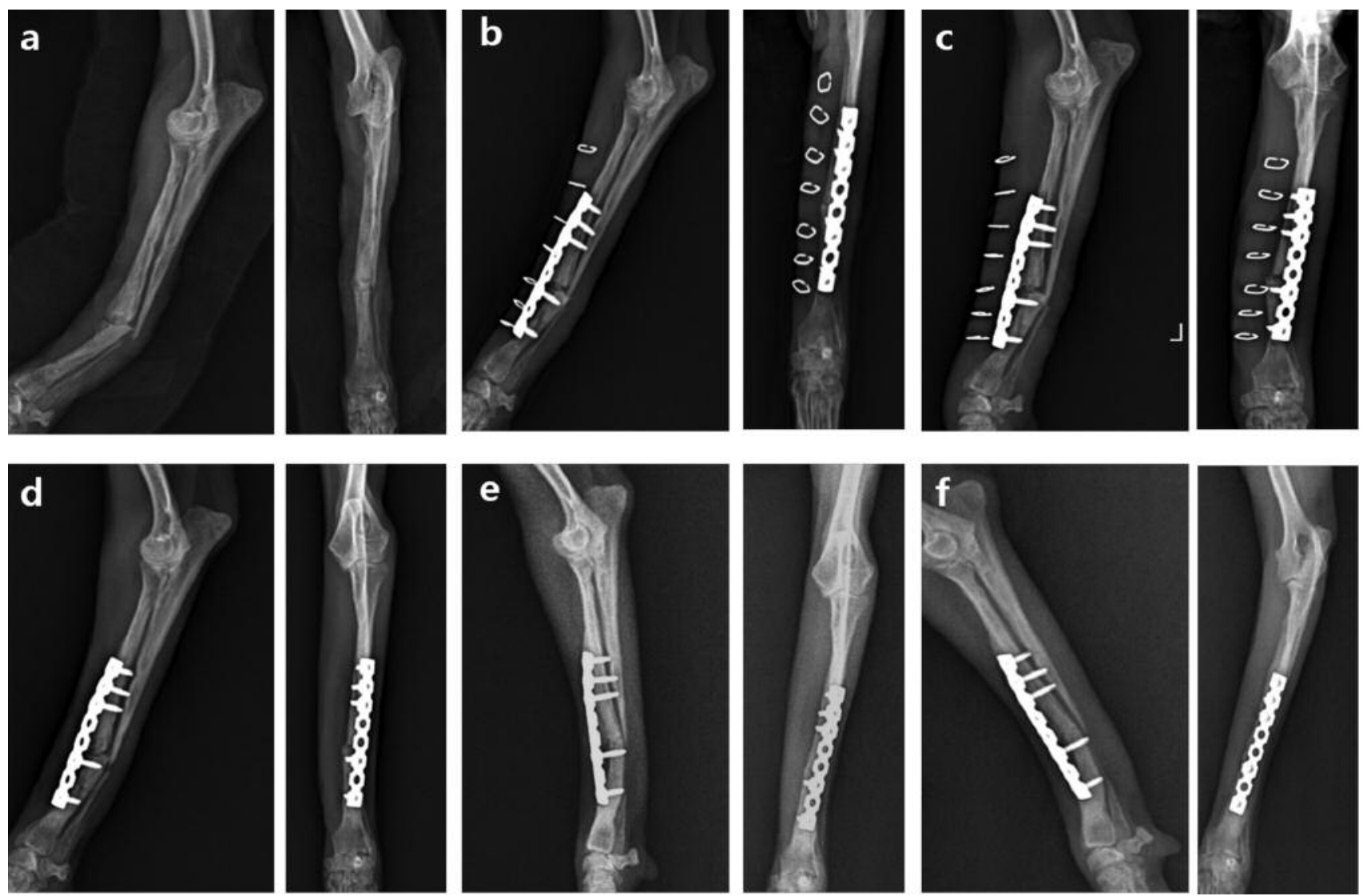

Figure 4. The bone healing process after application of the plate and cryoGelMA-BMP-2 at the fractured site (Lateral, Dorsoventral position). (a) A radiograph showing the site of the lesion four months after the fracture. Bone opacity decreased, and the shaft was irregular, with the presence of the fractured line. (b) A radiograph showing the treatment site post operation. Application of cryoGelMA-BMP-2 between the plate and the radius. As tissue fibrosis was evident, the reduction was not completely accurate. (c) Radiograph showing the treatment site a week after the operation. The margin of the distal fragment was slightly convex. (d) Radiograph showing the bone healing reaction, and narrowing of the fracture line 3 weeks post-operation. (e) Seven weeks after the operation, narrowing of the fractured space was observed. (f) Two months after surgery, the fractured fragments were successfully united.

biomolecules is mediated by either a change in GelMA concentration or methacrylation. The traditional type of gelatin has a lower ability of control over the release speed (18). The glutaraldehyde microparticles are cytotoxic, leading to the possibility of calcification and inflammation. It was reported that the incorporation of BMP-2 into GelMA hydrogel had relatively better efficacy and safety compared to the direct application of BMP-2 and glutaraldehyde BMP$2(19-21)$.

A previous study reported that the duration of BMP-2 release was 2 weeks for collagen hydrogel, 4 weeks for gelatin hydrogel, and about 7 weeks for GelMA-BMP-2 (17, 22). Nonetheless, the duration of a controlled release of BMP-2 with CryoGelMA has yet to be unidentified. The application of BMP-2, without a carrier, had a limited halflife of only few hours under in vitro conditions (23).
Moreover, it was shown that GelMA-BMP-2 was superior to other methods of delivery. Based on the duration of the controlled release of GelMA-BMP-2, we were able to predict the efficacy of cryoGelMA-BMP-2. We expected a longer maintenance period compared to that of GelMA because of its spacious accommodation of BMP-2.

In this case study, the bone healing process was shown to be active after a few weeks from the operation. The process of bone healing involved hardening of the tissue and swelling at the fracture site, thereby indicating the activation of BMP-2, which was released from cryoGelMA. Although excessive callus was generated after bone remodeling, the bone was functionally and structurally returned to its premorbid state. A previous study showed that GelMA was able to maintain effective delivery of BMP-2 under in vitro condition (18). However, there has been no evaluation of 
cryoGelMA-BMP-2 efficacy under in vitro and in vivo conditions. CryoGelMA is a promising biomaterial because of its affordable costs, and practicality for delivering high concentrations of biomolecules (15). We demonstrated the efficacy of cryoGelMA-BMP-2 treatment in two dogs. CryoGelMA-BMP-2 was applied only once during each surgical procedure and the bone healing capacity was shown to be sufficient to enhance bone recovery. Although we did not compare the efficacy of GelMA and cryoGelMA, previous literature has shown that cryoGelMA is a more effective carrier of BMP-2. In addition, CryoGelMA has the existing advantages of the GelMA biomaterial. For these reasons, cryoGelMA is a potentially effective biomaterial for accelerated bone recovery, and for the continued, slow delivery of BMP-2.

This case study had several limitations including the small case number, lack of control groups, and its retrospective nature. We needed to set up a control group to compare among CryoGelMA-BMP-2, GelMA-BMP-2, BMP-2 only group and a group with saline to distinguish the efficacy of each material, we could not set up the control group due to lack of the number of case. Furthermore, only radiology and observation-based walking analysis were used as evaluation matters in these two cases. However, we need to supplement our method of evaluation with computed gait analyzer which is for quantifying the result, and it is applicable as an objective assessment. Weight bearing with force plate could be another acceptable option. There is necessity to apply these methods for the further cases.

This reasonably priced biomaterial would be offered to patients who require treatment for nonunion, or delayedunion fracture. Although the loading concentration and specific duration of BMP-2 delivery via cryoGelMA have yet to be evaluated, cryoGelMA-BMP-2 is a valuable, efficacious material for treating nonunion or delayed-union injuries.

\section{Acknowledgements}

This study was supported by the Basic Science Research Program through the National Research Foundation of Korea (NRF) funded by the Ministry of Science, ICT \& Future Planning (NRF2015R1C1A1A01051759).

\section{References}

1 Nakashima M: Induction of dentine in amputated pulp of dogs by recombinant human bone morphogenetic proteins- 2 and- 4 with collagen matrix. Arch Oral Biol 39(12): 1085-1089, 1994.

2 Okamoto T, Yamamoto Y, Gotoh M, Huang CL, Nakamura T, Shimizu Y, Tabata Y and Yokomise H: Slow release of bone morphogenetic protein 2 from a gelatin sponge to promote regeneration of tracheal cartilage in a canine model. J Thorac Cardiovasc Surg 127(2): 329-334, 2004.
3 Gerstenfeld LC, Cullinane DM, Barnes GL, Graves DT and Einhorn TA: Fracture healing as a post-natal developmental process: Molecular, spatial, and temporal aspects of its regulation. J Cell Biochem 88(5): 873-884, 2003.

4 Lieberman JR, Le LQ, Wu L, Finerman GA, Berk A, Witte ON and Stevenson S: Regional gene therapy with a BMP-2producing murine stromal cell line induces heterotopic and orthotopic bone formation in rodents. J Orthop Res 16(3): 330339, 1998.

5 Murakami N, Saito N, Takahashi J, Ota H, Horiuchi H, Nawata M, Okada T, Nozaki K and Takaoka K: Repair of a proximal femoral bone defect in dogs using a porous surfaced prosthesis in combination with recombinant BMP-2 and a synthetic polymer carrier. Biomaterials 24(13): 2153-2159, 2003.

6 Bostrom MP: Expression of bone morphogenetic proteins in fracture healing. Clin Orthop Relat Res 355: S116-S123, 1998.

7 Dai KR, Xu XL, Tang TT, Zhu ZA, Yu CF, Lou JR and Zhang XL: Repairing of goat tibial bone defects with BMP-2 genemodified tissue-engineered bone. Calcif Tissue Int 77(1): 55-61, 2005.

8 Asamura S, Mochizuki Y, Yamamoto M, Tabata Y and Isogai N: Bone regeneration using a bone morphogenetic protein-2 saturated slow-release gelatin hydrogel sheet: evaluation in a canine orbital floor fracture model. Ann Plast Surg 64(4): 496502, 2010.

9 Patel ZS, Yamamoto M, Ueda H, Tabata Y and Mikos AG: Biodegradable gelatin microparticles as delivery systems for the controlled release of bone morphogenetic protein-2. Acta Biomater 4(5): 1126-1138, 2008.

10 Yamamoto M, Ikada Y and Tabata Y: Controlled release of growth factors based on biodegradation of gelatin hydrogel. J Biomater Sci Polym Ed 12(1): 77-88, 2001.

11 Young S, Wong M, Tabata Y and Mikos AG: Gelatin as a delivery vehicle for the controlled release of bioactive molecules. J Control Release 109(1-3): 256-274, 2005.

12 Holland TA, Tabata Y and Mikos AG: Dual growth factor delivery from degradable oligo (poly (ethylene glycol) fumarate) hydrogel scaffolds for cartilage tissue engineering. J Control Release 101(1-3): 111-125, 2005.

13 Solorio LD, Dhami CD, Dang PN, Vieregge EL and Alsberg E: Spatiotemporal regulation of chondrogenic differentiation with controlled delivery of transforming growth factor- $\beta 1$ from gelatin microspheres in mesenchymal stem cell aggregates. Stem Cells Transl Med 1(8): 632-639, 2012.

14 Solorio LD, Vieregge EL, Dhami CD, Dang PN and Alsberg E: Engineered cartilage via self-assembled hMSC sheets with incorporated biodegradable gelatin microspheres releasing transforming growth factor- $\beta 1$. J Control Release 158(2): $224-$ 232, 2012.

15 Henderson TM, Ladewig K, Haylock DN, McLean KM and O'Connor AJ: Cryogels for biomedical applications. J Mater Chem B 1: 2682-2695, 2013.

16 Hoch E, Schuh C, Hirth T, Tovar GE and Borchers K: Stiff gelatin hydrogels can be photo-chemically synthesized from low viscous gelatin solutions using molecularly functionalized gelatin with a high degree of methacrylation. J Mater Sci Mat Med 23(11): 2607-2617, 2012.

17 Nguyen AH, McKinney J, Miller T, Bongiorno T and McDevitt TC: Gelatin methacrylate microspheres for controlled growth factor release. Acta Biomater 13: 101-110, 2015. 
18 Samorezov JE, Headley EB, Everett CR and Alsberg E: Sustained presentation of BMP-2 enhances osteogenic differentiation of human adipose-derived stem cells in gelatin hydrogels. J Biomed Mater Res Part A 104(6): 1387-1397, 2016.

19 Bigi A, Cojazzi G, Panzavolta S, Rubini K and Roveri N: Mechanical and thermal properties of gelatin films at different degrees of glutaraldehyde crosslinking. Biomaterials 22(8): 763 768, 2001

20 Ruijgrok JM, De Wijn JR and Boon ME: Glutaraldehyde crosslinking of collagen: effects of time, temperature, concentration and presoaking as measured by shrinkage temperature. Clin Mater 17(1): 23-27, 1994.

21 Sung HW, Huang RN, Huang LL and Tsai CC: In vitro evaluation of cytotoxicity of a naturally occurring cross-linking reagent for biological tissue fixation. J Biomater Sci Polym Ed 10(1): 63-78, 1999.
22 Yamamoto M, Takahashi Y and Tabata Y: Controlled release by biodegradable hydrogels enhances the ectopic bone formation of bone morphogenetic protein. Biomaterials 24(24): 4375-4383, 2003.

23 Bramono DS, Murali S, Rai B, Ling L, Poh WT, Lim ZX, Stein GS, Nurcombe V, van Wiljnen AJ and Cool SM: Bone marrowderived heparan sulfate potentiates the osteogenic activity of bone morphogenetic protein-2 (BMP-2). Bone 50(4): 954-964, 2012.

Received January 16, 2018

Revised February 7, 2018

Accepted February 8, 2018 